\author{
(с) Э.А. Янар*, Н.В. Маказан, Е.М. Орлова, М.А. Карева
}

Национальный медицинский исследовательский центр эндокринологии, Москва, Россия

Болезнь Иценко-Кушинга (БИК) - редкое мультисистемное заболевание, причиной которого является АКТГ-секретирующая аденома гипофиза (кортикотропинома), обуславливающая гиперсекрецию кортизола пучковой зоной коры надпочечников.

БИК у детей может быть обусловлена как соматическими, так и герминативными мутациями, последние обуславливают развитие синдромов множественных эндокринных опухолей. При отсутствии лечения БИК приводит к тяжелой инвалидизации и смертельно-опасным осложнениям. В настоящее время первоочередным методом лечения является хирургическое удаление аденомы, в результате которого могут развиться пери- и постоперационные осложнения и сохраняется возможность повторных вмешательств. В то же время результаты исследований молекулярно-генетических механизмов формирования кортикотропином, от нарушения регуляции клеточного цикла и апоптоза до нарушений регуляции гипоталамо-гипофизарной-надпочечниковой оси, открывают возможности таргетной лекарственной терапии при БИК. Возможность наличия множественных эндокринных опухолей при БИК и перспективы таргетной терапии делают понимание причин и механизмов образования кортикотропином важным аспектом квалификации практикующих детских эндокринологов.

В настоящем обзоре отображены современные представления о молекулярно-генетических аспектах формирования кортикотропином и возможностях их лекарственной терапии.

КЛЮЧЕВЫЕ СЛОВА: гиперкортицизм; болезнь Иценко-Кушинга; кортикотропинома; аденома гипофиза.

\title{
GENETIC BASIS OF CUSHING'S DISEASE IN CHILDREN AND TARGETED THERAPEUTIC FUTURE PERSPECTIVES
}

\author{
(c) Eda A. Yanar*, Nadezhda V. Makazan, Elizaveta M. Orlova, Maria A. Kareva
}

Endocrinology Research Centre, Moscow, Russia

Cushing's disease (CD) is a multisystem disorder of a cortisol excess caused by ACTH -secreting pituitary tumor (corticotropinoma). $C D$ in children is due to somatic or germline mutations with the late onset causing multiple endocrine tumors. If not treated, hypercortisolism leads to severe decrease in quality of life and life-threating conditions. The first-line treatment for $C D$ is pituitary surgery, which might be followed by complications and relapse with necessity of additional surgery or initiations of second-line treatment. Recent studies of molecular basis of corticotropinoma development made it possible to employ medical therapy in CD. Understanding of corticotropinoma etiology and pathogenesis is an important part of education for pediatric endocrinologists since we need to keep in mind possibility of multisystem disorder in case of CD in children and because medical therapy might gain more important role for CD treatment in future.

The most actual genetic aspects of corticotroph adenomas growth and the medical treatment opportunities are present in this review.

KEYWORDS: Cushing Syndrome; Pituitary ACTH Hypersecretion; ACTH-Secreting Pituitary Adenoma.

\section{ВВЕДЕНИЕ}

Эндогенный гиперкортицизм - тяжелое мультисистемное заболевание, причиной которого является хроническая гиперпродукция гормона коры надпочечников - кортизола. Частота возникновения новых случаев заболевания - 0,7-2,4 на миллион людей ежегодно, и только $10 \%$ из них приходится на детское население [1].

Как в педиатрической, так и во взрослой практике кортикотропинома является самой частой причиной эндогенного гиперкортицизма, составляя 75-80\% случаев, при этом у детей она чаще встречается в возрасте старше 6 лет [2, 3]. Среди гормонально-активных аденом гипофиза кортикотропиномы составляют 4-8\% [4].

Для понимания причин, лежащих в основе развития кортикотропином, важно иметь представление о молекулярно-генетических механизмах регуляции гипоталамо-гипофизарно-надпочечниковой оси в норме.

Регуляция гипоталамо-гипофизарно-надпочечниковой оси

Секреция адренокортикотропного гормона (АКТГ) регулируется по принципу прямой связи под влиянием кортикотропин-рилизинг-гормона (КРГ) и вазопрессина и отрицательной обратной связи под влиянием глюкокортикоидов (ГК). 
В кортикотрофах аденогипофиза КРГ и вазопрессин связываются со своими рецепторами - КРГ-рецептором 1 типа (КРГр1) и рецептором к вазопрессину 1b типа (V1bR), что приводит к новому синтезу АКТГ из проопиомеланокортина (ПОМК) и высвобождению раннее синтезированного АКТГ [5].

Так как КРГр1 является трансмембранным рецептором и не способен самостоятельно воздействовать на ДНК, его эффект опосредуется медиаторами и транскрипторными факторами, взаимодействующими в ядре с промоторными областями гена POMC [5]. К этим медиаторам относятся фактор роста нервов IB (nerve growth factor IB, NGFI-B или Nur77), ряд ядерных рецепторов, среди которых - тестикулярные ядерные рецепторы 4 типа (Testicular orphan nuclear receptor-4, TR4), коактиватор стероидного рецептора-2 (steroid receptor coactivator-2, SRC-2), регуляторные элементы Pitx/Tpit и NeuroD1 [6, 7]. После активации транскрипторные факторы связываются с соответствующими элементами промоторной области гена $P O M C$, запускают транскрипцию гена, что ведет к синтезу ПОМК и в дальнейшем - образованию его конечных продуктов, среди которых - АКТГ [5].

В коре надпочечников АКТГ связывается с клетками с помощью меланокортинового рецептора 2 типа (MCR2), как следствие - активируется стероидогенез с образованием Гк.

Рецепторы к Гк (ГкР) расположены практически во всех ядерных клетках организма. В неактивном состоянии ГкР находятся в цитоплазме в комплексе с молекулярными шаперонами, в том числе с белком теплового шока 90 (HSP90), которые обеспечивают рецептору нужное для связи с Гк конформационное состояние и защищают его от деградации [8]. В активном состоянии, после связывания с Гк, ГкР поступает в клеточное ядро. Будучи транскрипционным фактором, активированный ГкР изменяет экспрессию генов в клетках-мишенях: инициирует транскрипцию генов напрямую, связываясь со специальными участками ДНК - ДНК-полидромными и Гк-реагирующими элементами, и подавляет транскрипцию преимущественно за счет непрямого воздействия на ДНК через белки-транскрипционные факторы (транс-репрессия) [9].

Способность активированных ГкР подавлять транскрипцию генов является осново работы механизма отрицательной обратной связи: КРГ стимулирует синтез ПОМК, что ведет к повышению уровня Гк крови, а Гк, в свою очередь, подавляют синтез КРГ и ПОМК через ГкР [10].

В механизме подавления транскрипции РОМС важную роль играют транскрипционные ко-регуляторы - Brg1 (Brachma-related gene 1) и HDAC2 (Histone deacetylases, гистон-деацетилаза 2 типа). При участии Brg1 активированный ГкР связывается с NGFI-B (Nur77) и HDAC2, образуя комплекс, подавляющий транскрипцию РОМС [9].

Помимо Гк, дофамин и соматостатин также способны снижать уровень АКТГ крови за счет ингибирования его секреции [11].

Инактивация Гк в клетках-мишенях происходит за счет фермента 11-бета-гидроксистероиддегидрогеназы (11ßHSD). У этого фермента есть 2 изоформы: 1-й тип - 11ßHSD катализирует образование кортизола из кортизона, а 2-й тип - 11ßHSD, наоборот, способствует деградации кортизола в кортизон [10].

Схема регуляции гипоталамо-гипофизарно-надпочечниковой оси представлена на рисунке 1.
Молекулярно-генетические основы образования кортикотропином: соматические мутации

Как и другие аденомы гипофиза, кортикотропиномы происходят из нормальных кортикотрофов аденогипофиза, являясь по своей природе моноклональными опухолями, т.е. происходящими из одной клетки [5]. В ядре кортикотрофной клетки в одном из генов первично возникают соматические мутации, приводящие к повышенной функции и неконтролируемому делению этой клетки [5]. Нормальные кортикотрофы при этом начинают тормозить собственный рост в ответ на повышенные уровни Гк, что приводит к преимущественному росту мутантных кортикотрофов [12].

На протяжении более 20 лет ведутся исследования по идентификации этапов регуляции гипоталамогипофизарно-надпочечниковой оси и клеточного роста, нарушения в которых приводят к образованию кортикотропином. Анализ данных этих исследований позволяет выделить несколько возможных механизмов формирования кортикотропином (рис. 2).

Нарушение механизма отрицательной обратной связи: глюкокортикоидный рецептор и репрессия синтеза ПОМК

Ключевой фактор в работе отрицательной обратной связи для гипоталамо-гипофизарно-надпочечниковой оси - это ГкР, в связи с чем логично было бы отвести ему ведущую роль в развитии кортикотропином. Тем не менее мутации в гене ГкР (NR3C1) при болезни Иценко-Кушинга (БИК) описаны лишь в единичных случаях $[10,13]$. Относительно более частым нарушением ГкР может являться снижение его активности за счет соматической потери одного аллеля гена NR3C1 в клетках опухоли (потеря гетерозиготности) - такое нарушение было выявлено в исследовании Huizenga N.A. et al. в 6 случаях из 22 кортикотропином [14].

Повышенная экспрессия белка теплового шока 90 (HSP90) также может играть роль в нарушении механизмов отрицательной обратной связи: гиперпродукция HSP90 приводит к нарушению нормального конформационного состояния ГкР, теряется аффинность ГкР к Гк и, следовательно, возможность оказывать ингибирующий эффект на транскрипцию гена РОМС [10].

В потере отрицательной обратной связи при кортикотропиномах также могут играть роль транскрипционные ко-регуляторы, без которых ГкР не способен подавить транскрипцию POMC: Brg1 и $\operatorname{HDAC2}[9,10]$. В исследовании на клеточных моделях было продемонстрировано, что в отсутствие Brg1 активность промотера РОМС снижается на 50\%, а оставшаяся активность перестает подавляться в ответ на дексаметазон [9]. Однако результаты исследований ДНК, полученных из кортикотропином, на мутации в генах, кодирующих Brg1 и HDAC2, не подтверждают эту гипотезу $[15,16]$. Данные результаты не позволяют однозначно судить о вкладе Brg1 и HDAC2 в развитие кортикотропином.

\section{Нарушение механизма прямой связи: активация синтеза} ПОМК

Рецептор к КРГ является трансмембранным, поэтому после его активации в кортикотрофе включается внутриклеточный путь передачи сигнала. На последних этапах этого пути активируются транскрипционные факторы: 


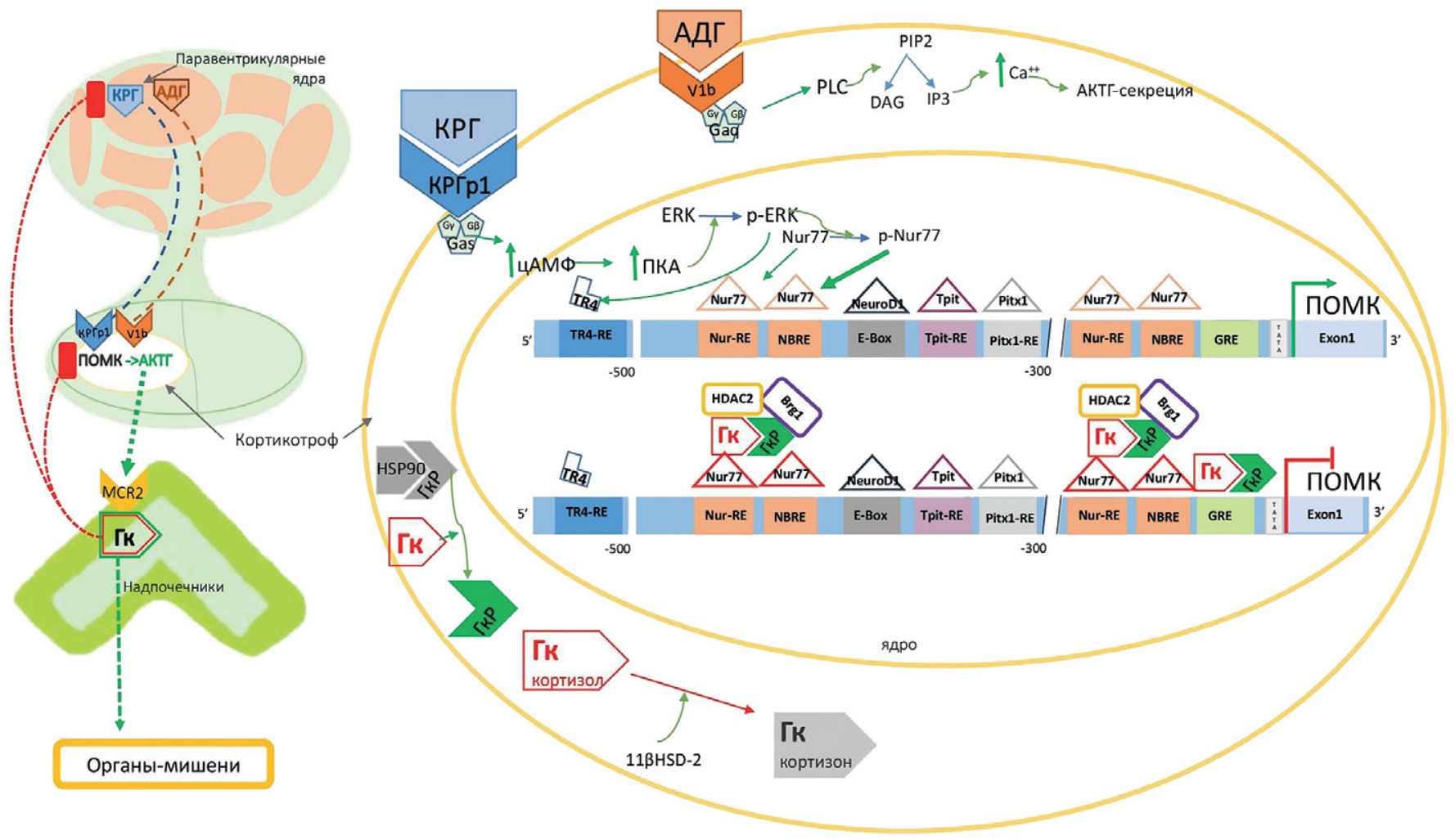

Рисунок 1. Схема регуляции гипоталамо-гипофизарно-надпочечниковой оси и синтеза проопиомеланокортина.

Связывание антидиуретического гормона (АДГ) с его рецептором (рецептором к вазопрессину $1 \mathrm{~b}$ типа - V1bR) приводит к активации альфа-q-субъединицы G-белка (Gaq), что активирует фосфолипазу C (PLC), которая гидролизует фосфатидилинозитол (PIP2) до инозитолтрифосфата (IP3) и приводит к высвобождению Саa и стимуляции секреции адренокортикотропного гормона (АКТГ).

Связывание кортикотропин-рилизинг-гормона (КРГ) с его рецептором (КРГр1) приводит к активации стимулирующей альфа-субъединицы G-белка (Gas), что приводит к увеличению концентрации циклического аденозинмонофосфата (цАМФ), который активирует протеинкиназу A (ПКА). ПКА повышает активность транскрипционных факторов (фактора роста нервов IB (Nur77 $\rightarrow$ p-Nur), тестикулярных ядерных рецепторов 4 типа (TR4)) через фосфорилирование ERK (extracellular signal-regulated kinase), которая потенцирует их связывание с чувствительными элементами промоторной области гена POMC (регуляторных элементов Pitx/Tpit и NeuroD1) и запускает транскрипцию, что ведет к стимуляции синтеза проопиомеланокортина (ПОМК).

В коре надпочечников АКТГ связывается с клетками с помощью меланокортинового рецептора (MCR2), активируя стероидогенез с образованием глюкокортикоидов (ГК).

По механизму отрицательной обратной связи Гк блокируют синтез и секрецию КРГ и ПОМК в гипоталамусе и аденогипофизе соответственно.

В отсутствие Гк в кортикотрофах глюкокортикоидный рецептор (ГкР) находится в неактивном состоянии в комплексе с белком теплового шока 90 (HSP90), который защищает его от деградации. В активном состоянии, после связывания с Гк, ГкР поступает в клеточное ядро. Активированный ГКР связывается напрямую с глюкокортикоид-реагирующими элементами (GRE), а также формирует комплексы c Brg1 (Brachma-related gene 1), HDAC2 (гистон-деацетилаза 2) и с Nur77, подавляя транскрипцию РОМС.

Инактивация Гк в клетках-мишенях происходит за счет фермента 11-бета-гидроксистероиддегидрогеназы (11ßHSD), который способствует деградации кортизола в кортизон.

они проникают в ядро и связываются с промоторной областью РОМС, обеспечивая начало его транскрипции [10].

Среди этих транскрипционных факторов возможная роль в развитии кортикотропином была оценена для фактора роста нервов IB (nerve growth factor IB, NGFI-B, или Nur77) и тестикулярного ядерного рецептора 4 типа (testicular orphan nuclear receptor-4, TR4) [10].

Как показали результаты исследования Li Du et al. (2013), из 12 образцов кортикотропином в 10 случаях экспрессия TR4 в повышенном значении определялась в клеточных ядрах, тогда как в кортикотрофах нормальных гипофизов (5 аутопсийных образцов) экспрессия была выражена меньше и отмечалась только в цитоплазме клеток [7]. Помимо непосредственной инициации транскрипции гена POMC, TR4 также способен связываться с ГкР и блокировать негативный эффект ГкР на транскрипцию РОМС [7].

В исследовании Tabuchi Y. et al. (2016) была выявлена значимо повышенная экспрессия Nur77 в образцах кортикотропином от пациентов с БИК $(n=7)$ по сравнению с данными от кортикотропином пациентов с субклинической БИК* $(\mathrm{n}=6)$, и функционально неактивных аденом гипофиза $(n=10)$, что также может указывать на значение этого транскрипционного фактора в развитии кортикотропином [17].

\section{Нарушение метаболизма кортизола}

Помимо нарушений регуляции синтеза и секреции ПОМК и АКТГ, при кортикотропиномах может иметь значение метаболизм кортизола. Упомянутое выше исследование Tateno T. et al. (2007) показало, что в кортико-

\footnotetext{
* Субклиническая БИК диагностировалась авторами статьи на основании отсутствия клинических проявлений гиперкортицизма при лабораторных критериях, свидетельствующих о наличии АКТГ-секретирующей кортикотропиномы (нарушение циркадного ритма, отсутствие супрессии кортизола на тестах с дексаметазоном, наличие аденомы гипофиза по данным МРТ) [17].
} 


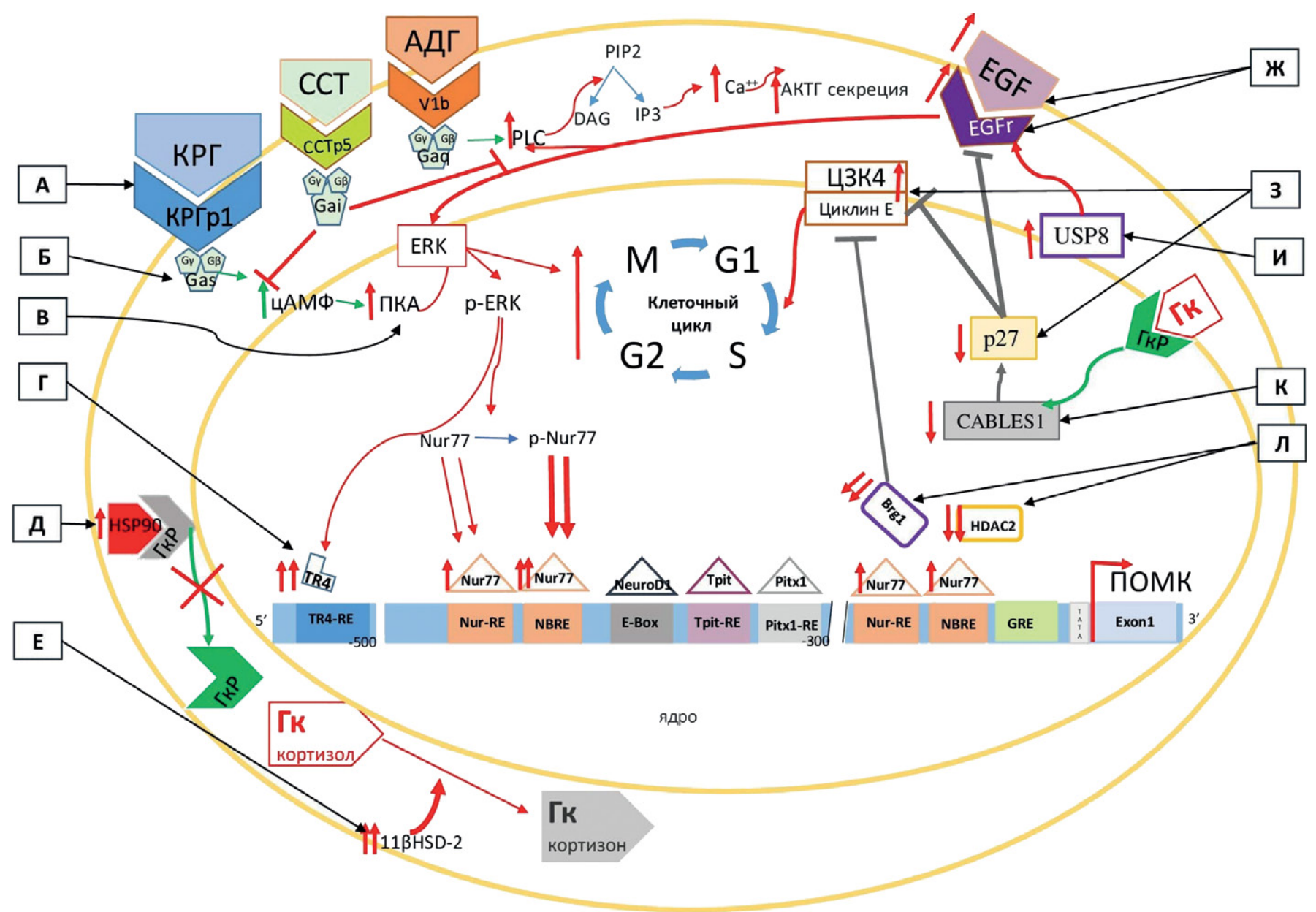

Рисунок 2. Нарушения механизмов отрицательной обратной связи, регуляции транскрипции РОМС и клеточной пролиферации, описанные при болезни Иценко-Кушинга.

А: Инактивирующие мутации в гене глюкокортикоидного рецептора (ГКР - NR3C1) приводят к резистентности к глюкокортикоидам (Гк) и гиперпродукции проопиомеланокортина (ПОМК).

Б: Активирующие соматические мутации в гене GNAS запускают передачу сигнала от рецептора кортикотропин-рилизинг-гормона 1 типа (КРГр1) $\rightarrow$ повышается синтез адренокортикотропного гормона (АКТГ).

B: Инактивирующая мутация в гене PRKAR1A перестает ограничивать деятельность каталитической субъединицы ПКА $\rightarrow$ повышение синтеза АКТГ.

Г: Тестикулярный ядерный рецептор 4 типа (TR4) является одним из факторов транскрипции гена РОМС, его повышенная экспрессия приводит к гиперпродукции ПОМК.

Д:Повышенная экспрессия белка теплового шока 90 (HSP90) приводит к нарушению нормального конформационного состояния ГкР и его деградации потеря ингибирующего эффекта на транскрипцию гена РОМС.

E:Повышенный уровень экспрессии 11-бета-гидроксистероиддегидрогеназы $11 \beta \mathrm{HSD} 2$ может приводить к ускоренной деградации кортизола и развитию парциальной глюкокортикоидной резистентности.

ж: Рецептор эпидермального ростового фактора (EGF) стимулирует сигнальные пути митоген-активируемых протеинкиназ (MAPK) и фосфолипазы C (PLC), инициирует клеточную пролиферацию, запускает механизмы антиапоптоза.

3: Снижение уровня р27 (ингибитора СDК) приводит к повышению экспрессии циклина Е, что вызывает ускорение репликации клеток.

И:При повышенной активности убиквитин-специфической протеазы 8 (USP8) стимулируется функция EGF, что приводит к повышенной экспрессии POMC. К:Инактивирующие мутации в гене CABLES1 повышают экспрессию р27, который угнетает рецепторы к эпидермальному ростовому фактору (EGF) и блокирует клеточную пролиферацию.

Л: Активный глюкокортикоидный рецептор (ГкР) ингибирует транскрипцию POMC в комплексе с HDAC2 (гистон-деацетилаза 2) и Brg1 (Brachma-related gene 1). Brg1 подавляет активность циклина Е и CDK2 и негативно регулирует клеточную пролиферацию. Сниженная экспрессия HDAC2 и Brg1 нарушает формирование комплексов, подавляющих транскрипцию РОМС, и приводит к гиперпродукции ПОМК.

Изображения, представленные на рисунках: $Т$ указывают на активирующее влияние $Т$ указывают на ингибирующее влияние

тропиномах может отмечаться повышенный уровень экспрессии фермента, катализирующего переход кортизола в кортизон, 11-бета-гидроксистероиддегидрогеназы 2 типа (11ßHSD2) [15]. Также повышение 11ßHSD2 в 7 из 9 кортикотропином было отмечено ранее в исследовании Korbonits M. et al. (2007) [18]. Данные результаты свидетельствуют о том, что $11 \beta \mathrm{HSD} 2$, регулируя внутриклеточное содержание кортизола, может играть роль в развитии парциальной глюкокортикоидной резистентности при БИК.

\section{Нарушение регуляции клеточного чикла}

В жизненном цикле клетки выделяют несколько этапов развития. После появления клетка находится в пресинтетическом периоде развития (G1), во время которого происходит ее рост, синтез белков, образование органелл. Это самый длительный период в жизни клетки, после которого один за другим следуют периоды подготовки к делению и собственно деление. В синтетический период (S) происходит удвоение ДНК, после чего клетка вступает в период подготовки к делению (G2), во время 
которого она растет, идет синтез аденозинтрифосфата (АТФ), удваиваются органеллы. После подготовки в клетке происходит удвоение ядра и цитоплазмы (М). Еще выделяют фазу G0, когда клетка вступает в дифференцировку и к обычному циклу уже не возвращается [19]. При переходе из одного периода в другой клетка проходит определенные точки проверки, определяющие, будет ли она вовлечена в следующий период развития или нет [20].

Переход клетки из одной фазы в другую обеспечивается циклин-зависимыми киназами (CDK, cyclindependent kinases) и их активаторами - циклинами, в то время как приостановление смены периодов жизни клетки происходит под влиянием ингибиторов, нейтрализующих активность циклинов и CDK [19]. Равновесие между двумя этими процессами обеспечивает нормальное прохождение клеточного цикла.

Переход от G1 к S опосредуется циклином Д1 и связанной с ним CDK4, а также циклином Е в комплексе с CDK2 $[19,20]$. Гиперэкспрессия этих циклинов играет важную роль в онкогенезе, так как она приводит к ускоренному переходу к репликации неготовых клеток с недостаточным запасом факторов роста [20]. В исследовании Jordan S. (2000), посвященном оценке роли циклинов Д и Е в формировании различных видов опухолей гипофиза (95 образцов), было выявлено, что во всех видах опухолей, но особенно в кортикотропиномах $(n=19)$ отмечалась повышенная экспрессия циклина $\mathrm{E}$ [20]. По всей видимости, такое повышение циклина Е в кортикотропиномах связано со сниженным содержанием в этих опухолях супрессора опухолевого роста - ингибитора циклинзависимой киназы 1В - p27, в функции которого как раз входит блокирование CDK $[10,19]$. Помимо р27, способностью подавлять активность циклина Е и CDK2 обладает транскрипционный ко-регулятор - Brg1: в исследовании Roussel-Gervais A. (2010) клеточной модели кортикотрофов было показано, что при отсутствии Brg1 отмечается значимое повышение экспрессии циклина E [21]. Таким образом, снижение активности Brg1 в кортикотрофах может приводить как к нарушению отрицательной обратной связи, так и повреждению клеточного цикла.

Еще одно доказательство тесной взаимосвязи между регуляцией отрицательной обратной связи и клеточного цикла получено в другом исследовании Roussel-Gervais A. (2016), где было показано, что в кортикотрофах Гк вызывают активацию гена CABLES1. Продукт этого гена является негативным регулятором клеточного цикла: в его отсутствие в клеточных моделях кортикотрофов отмечалось резкое повышение пролиферирующей активности и снижение экспрессии р27 [22]. Данные о нарушениях CABLES1 в клетках кортикотропином человека варьируют: в исследовании Hernández-Ramírez L.C. (2017) потенциально патогенные мутации в гене CABLES1 были выявлены в 2\% случаев $(n=181)$ [23], по данным Roussel-Gervais A. $(2016, n=31)$, снижение экспрессии CABLES1 обнаружено в 55\% случаев [22]. Согласно Roussel-Gervais A. (2016), CABLES1 повышает экспрессию р27, который, в свою очередь, блокирует клеточную пролиферацию за счет угнетения рецепторов к эпидермальному ростовому фактору (EGF), хорошо известному своей онкогенной активностью $[10,13]$.

Во многих опухолях, в том числе и в кортикотропиномах, отмечается повышенная экспрессия как самого EGF, так и рецепторов к нему [5]. Стимулируя тирозинкиназную активность, активированный рецептор к EGF инициирует клеточную пролиферацию, адгезию и инвазию трансформированных клеток, запускает механизмы антиапоптоза [10]. В исследовании Theodoropoulou M. (2004) мРНК рецептора EGF была найдена в 2 кортикотропиномах из 49 исследованных аденом гипофиза, тогда как из доступных для иммуноанализа 102 образцов ткани аденом гипофиза наибольшее количество иммунопозитивных к рецептору EGF случаев пришлось на кортикотропиномы (50\% от всех кортикотропином) [24]. Высокая экспрессия рецептора EGF в кортикотропиномах предполагает, что этот рецептор может стать кандидатом для терапевтического лечения в этих аденомах гипофиза.

Помимо EGF, в формировании кортикотропином может иметь значение также другой ростовой фактор фактор роста фибробластов 4 типа (FGFR4), выявляемый, согласно данным Qian Z.R. (2004), в 50\% случаев кортикотропином [25].

B 2015 г. Reincke М. опубликовал исследование, в котором с помощью экзомного секвенирования ДНК, выделенной из ткани 10 удаленных кортикотропином, в 4 случаях были выявлены соматические мутации в гене USP8, который кодирует убиквитин-специфическую протеазу 8 [26]. Для нормальной жизнедеятельности клетки важное значение имеет своевременная деградация дефектных белков. Разрушение таких белков (протеолиз) производится протеосомой. Запуск протеолиза начинается после того, как к белку-мишени присоединяется убиквитин, и такой комплекс распознается протеосомой, вызывая разрушение белка. Такой путь деградации белков может быть предотвращен, так как комплекс убиквитин-белок-мишень способен разрушаться под влиянием специальных ферментов - убиквитин-специфических протеаз (USP) $[10,27]$. Из этих ферментов в кортикотрофах особую роль играет убиквитин-специфическая протеаза 8 (USP8), так как она регулирует внутриклеточное содержание рецепторов EGF [27]. Активирующие мутации USP8 стабилизируют и предотвращают деградацию рецепторов EGF, стимулируя тем самым функцию EGF в кортикотрофах и приводя к повышенной экспрессии РОМС $[10,13]$. В последующих исследованиях выяснилось, что из всех соматических мутаций активирующие мутации USP8 в кортикотропиномах встречаются с наибольшей частотой (31-61\%) как среди взрослых пациентов, так и среди детей. Соматические мутации USP8 ассоциированы с формированием микроаденом с высокой секрецией АКТГ и повышенным риском рецидивов $[10,13,28,29]$. Также, по результатам работы Hayashi et al., в кортикотропиномах, несущих мутацию в гене USP8, чаще выявляются рецепторы к соматостатину 5 типа, в связи с чем они могут отвечать на терапию препаратами аналогов соматостатина [28]. Совсем недавно, в 2019 г., появилось описание нового генетического синдрома, вызванного герминативной мутацией в гене USP8 у девочки 16 лет с рецидивирующей БИК в совокупности с задержкой умственного развития, ихтиозоформной эритродермией, хронической почечной и легочной недостаточностью, гипергликемией, кардиомиопатией и парциальным дефицитом соматотропного гормона (СТГ) [30].

Другие соматические мутации выявляются при кортикотропиномах реже. Одной из них является мутация в гене TP53 $[10,13]$. Белок, кодируемый геном TP53, является ключевым опухолевым супрессором, участвующим 
в регуляции клеточного цикла, репарации ДНК, апоптозе. Мутации ТР53 приводят к нарушению структуры и функции белка р53 при многих видах опухолей [31]. В исследовании Buckley N. et al. (1995), проведенном на 95 образцах аденом гипофиза и 26 инвазивных опухолях гипофиза, повышенное содержание р53 было выявлено в 2 из 4 инвазивных кортикотропином и в 16 из 32 неинвазивных кортикотропином [31]. Есть также описания 2 случаев АКТГ-секретирующих карцином гипофиза и 1 случай инвазивной кортикотропиномы с высоким индексом Кі-67, в которых были выявлены соматические мутации в гене ТP53 [13].

В 15\% случаев БИК в кортикотропиномах находят мутации в гене CTNN1B, кодирующем бета-катенин [32]. У мышей бета-катенин играет важную роль в стимуляции пролиферации эмбриональных кортикотрофов, его повышенная активация приводит к их гиперплазии [13]. Соматические мутации в CTNN1B встречаются также в 2/3 случаев функционально неактивных аденом гипофиза и 1/3 случаев адренокортикальных карцином, что не исключает возможности ассоциации этих мутаций с более агрессивным фенотипом опухолей [32].

В единичных случаях при аденомах гипофиза могут быть выявлены активирующие мутации в гене PIKЗСА, кодирующем каталитическую субъединицу р110а фосфатидил-инозитол-3'-киназы (РІ3К) [33]. РІ3К/АКТ-сигнальный путь играет важную роль в активации клеточного роста и пролиферации. Чаще всего нарушения PІКЗСА выявляются при раке молочной железы, колоректальном раке, раке эндометрия, тогда как при аденомах гипофиза они встречаются очень редко: есть описания случая инвазивной кортикотропиномы и случая неинвазивной АКТГсекретирующей микроаденомы гипофиза, в которых были выявлены мутации в гене PІКЗСА [33].

Очень редко при кортикотропиномах определялись соматические мутации в GNAS [13]. Ген GNAS кодирует стимулирующую альфа-субъединицу G-белка (Gas). G-белок необходим для передачи сигнала от трансмембранных гормональных рецепторов к клеточному ядру. Ряд гормонов, в том числе АКТГ, передает свое действие в клетки-мишени через Gas. Постзиготные мутации в GNAS вызывают развитие синдрома МакКьюна-Олбрайта-Брайцева, одним из редких компонентов которого является неонатальный синдром Кушинга за счет гиперплазии фетальной коры надпочечников [13]. При синдроме Мак-Кьюна-Олбрайта-Брайцева описаны гормонально-неактивные аденомы гипофиза, соматотропиномы и пролактиномы, при этом не описано развитие БИК вследствие кортикотропином. Однако в одном исследовании в трех случаях из 9 кортикотропином была выявлена соматическая мутация в GNAS [10, 13].

Молекулярно-генетические основы образования

кортикотропином: герминативные мутации

Подавляющее большинство аденом гипофиза как у детей, так и у взрослых являются спорадическими случаями, но менее чем в 5\% случаев они могут встречаться при ряде наследственных синдромов, что нужно учитывать при выявлении БИК у детей [10]. Особенностью детского возраста является относительно высокий по сравнению со взрослой популяцией удельный вес наследственных синдромов, включающих в свой состав кортикотропиномы.
Синдром множественных эндокринных неоплазий 1 muna (МЭН-1)

Синдром обусловлен мутацией в гене $M E N$, кодирующем белок менин, - опухолевый супрессор, который регулирует клеточный цикл и транскрипцию $[10,13]$. Недостаточность менина приводит к гиперплазии, а отсутствие менина - к опухолевой трансформации клеток. Наиболее часто развиваются опухоли паращитовидных желез, гастроэнтеропанкреатические нейроэндокринные опухоли (НЭО), и у 30-40\% пациентов выявляются аденомы гипофиза, из них только до 10\% случаев - кортикотропиномы $[34,35]$. В гипофизе менин взаимодействует с активином, активация которого приводит к снижению секреции пролактина (ПРЛ), СТГ и АКТГ и торможению клеточной пролиферации [36]. При синдроме МЭН-1 чаще встречаются макроаденомы (в 76-85\%), характеризующиеся более инвазивным ростом, однако случаи карцином крайне редки $[35,37]$. Также гиперкортицизм при синдроме МЭН-1 может быть АКТГ-независимого генеза [34].

Синдром множественных эндокринных неоплазий 4 muna (МЭН-4)

У 2\% пациентов с МЭН-1-подобным синдромом при отсутствии патологии в $M E N$ выявляются герминативные мутации в гене $C D K N 1 B$, в этом случае синдром называют МЭН-4 [10]. Продукт гена CDKN1B - супрессор опухолевого роста, ингибитор циклин-зависимой киназы 1В р27, который регулирует клеточный цикл. О роли р27 в развитии кортикотропином уже упоминалось выше: локальная сниженная экспрессия р27 отмечена в кортикотропиномах, что связывают с потерей функции его негативного регулятора CABLES [38]. В составе синдрома МЭН-4 - аденомы гипофиза, аденомы паращитовидных желез, нейроэндокринные опухоли, различные доброкачественные и злокачественные опухоли $[13,38]$. По данным исследования Chasseloup et al., в которое были включены 211 пациентов с БИК (из них 199 детей), было выявлено 5 детей, имеющих герминативную мутацию в гене CDKN1B (в 1 случае была также выявлена соматическая мутация в гене USP8) [38].

Синдром множественных эндокринных неоплазий

2-го muna (МЭН-2)

Классическими вариантами синдрома МЭН-2 являются сочетание медуллярного рака щитовидной железы с феохромоцитомой и гиперпаратиреозом (МЭН-2А) либо сочетание медуллярного рака щитовидной железы, феохромоцитомы, ганглионевром, утолщения зрительного нерва с марфаноподобной внешностью (МЭН-2Б) [10, 13]. Причиной возникновения синдрома служит гетерозиготная мутация в протоонкогене RET. Этот ген кодирует трансмембранный рецептор с тирозинкиназной активностью. RET активирует MTOR, отвечающий за рост, дифференцировку и выживание клетки. Аденомы гипофиза не являются классическим компонентом синдрома МЭН-2, описано лишь несколько случаев развития аденомы гипофиза в составе синдрома МЭН-2, среди которых у 3 пациентов была выявлена БИК, в двух из этих случаев доказана мутация в гене RET [39-41].

Синдром семейных изолированных аденом гипофиза

Аденомы гипофиза также могут встречаться в составе синдрома семейных изолированных аденом гипофиза (FIPA, familial isolated pituitary adenomas), характеризую- 
щегося появлением аденом гипофиза у 2 и более членов семьи. В 20\% семейных случаев причиной FIPA являются инактивирующие мутации в гене супрессора опухолевого роста AIP $[10,13]$. Белок AIP оказывает свой эффект при взаимодействии с арилуглеводородным рецептором. Описано лишь 3 случая кортикотропином у пациентов c FIPA и мутацией в AIP [42]. Большинство аденом у AIP-позитивных пациентов являются макроаденомами, в основном с инвазивным ростом, имеют более агрессивные характеристики и ассоциированы с более ранним началом заболевания и ускоренным ростом опухоли, резистентностью к консервативной терапии $[42,43]$.

\section{DICER-синдром}

Помимо МЭН и FIPA, аденомы гипофиза описаны также в составе DICER-синдрома, обусловленного гетерозиготными мутациями в гене DICER, кодирующем одноименный белок Dicer1. Dicer1 запускает процесс торможения экспрессии генов на посттранскрипционной стадии и деградацию ДНК при апоптозе, регулирует синтез малых РНК, также есть данные об участии Dicer1 в поддержании нормальной структуры и объема клеточного ядра [44]. Герминативные инактивирующие мутации в гене DICER1 приводят к развитию опухолевого синдрома с аутосомно-доминантным типом наследования с очень низкой пенетрантностью и ранней манифестацией. Проявления DICER-синдрома включают в себя пневмолегочные бластомы, кисты легких, кистозные нефромы, клеточные опухоли яичников Сертоли-Лейдига, мочеполовые саркомы, пинеобластомы, новообразования щитовидной железы и бластомы гипофиза $[10,13]$. Последние опухоли крайне редко встречаются, являются агрессивными, вероятно, врожденными опухолями. Все описанные случаи бластом гипофиза с герминативными и соматическими мутациями в DICER1 характеризовались АКТГ-зависимым гиперкортицизмом с манифестацией, начиная от первого года жизни, и крайне неблагоприятным прогнозом, в том числе летальным исходом, как описано в нескольких случаях у детей [13].

\section{Карни-комплекс}

Помимо мутаций онкогенов и генов-супрессоров опухолевого роста, к развитию множественных неоплазий приводит также патология внутриклеточных сигнальных путей, среди которых - нарушения цАМФ-зависимого сигнального пути [45]. Это каскадный путь передачи сигнала от активированного поверхностного рецептора к клеточному ядру: активация рецептора запускает работу G-белка, что, в свою очередь, приводит к повышению уровня цАМФ с последующей активацией протеинкиназы А и далее транскрипторных факторов, изменяющих экспрессию генов в соответствии с эффектом гормона. Рецептор-независимая активация любого из этапов этого пути приводит к повышенной клеточной функции, пролиферации и создает условия для развития опухолей [45]. Одним из звеньев этого пути является протеинкиназа А, действующая на уровне клеточного ядра. У протеинкиназы А (ПКА) сложное строение: она состоит из активной каталитической субъединицы и двух регуляторных, ограничивающих активность каталитической субъединицы в отсутствие цАМФ. Различают несколько подтипов регуляторной субъединицы (I-альфа, І-бета, II-альфа, II-бета), каждый из которых кодируется своим геном [46].
Инактивирующая мутация в гене регуляторной субъединицы І-альфа (PRKAR1A) приводит к приостановке ограничения деятельности каталитической субъединицы ПКА и повышению функциональной и пролиферативной активности клетки [47]. Клинически это приводит к развитию Карни-комплекса - аутосомно-доминантного мультисистемного заболевания, характеризующегося сочетанием МЭН, лентигиноза и миксом сердца $[10,13]$. Для Карникомплекса характерен синдром Кушинга на фоне двусторонней узелковой гиперплазии надпочечников, так как рецепторы к АКТГ в коре надпочечников проводят сигнал от АКТГ через цАМФ/ПКА-зависимый путь с вовлечением ПКА [46].

БИК при Карни-комплексе не характерна, хотя в один из путей передачи сигнала от рецепторов к КРГ к ядру тоже вовлечена ПКА. В настоящее время известно всего два случая БИК в сочетании с Карни-комплексом. В случае, описанном Kiefer F et al. (2017), у мужчины с Карни-комплексом (миксома, лентигиноз, Сертоли-клеточная опухоль) была обнаружена БИК, а в дополнение к герминативной мутации PRKAR1A в ткани удаленной кортикотропиномы была обнаружена частичная потеря аллеля на уровне, соответствующем гену PRKAR1A [48]. После удаления кортикотропиномы гиперкортицизм нивелировался и пациент оставался в ремиссии на протяжении 7 лет наблюдения. Авторы предполагали, что мутация в PRKAR1A сыграла роль в развитии кортикотропиномы в данном случае. Тем не менее полностью исключить коморбидность Карни-комплекса и БИК на фоне соматической потери всего локуса 17 хромосомы 17q24 тоже нельзя, так как потеря 17 хромосомы была описана в 6 из 11 случаев кортикотропином [48, 49].

Второй случай БИК при Карни-комплексе был опубликован Hernández-Ramírez L.C. et al. (2017) у мальчика, у которого в 15 лет была подтверждена БИК с успешным удалением кортикотропиномы, а спустя два года - вновь возник гиперкортицизм, уже АКТГ-независимый, на фоне двусторонней узелковой гиперплазии надпочечников. После выявления синдрома Кушинга ему было выполнено молекулярно-генетическое исследование и выявлена герминативная мутация в PRKAR1A, а также потеря гетерозиготности PRKAR1A в ткани кортикотропиномы с оставшейся мутантной аллелью [50].

\section{Таргетная терапия: текущие возможности}

и перспективы

Вопрос консервативной терапии поднимается в случае невыполнимости операции, ее неэффективности или в качестве предоперационной подготовки. Несмотря на достигнутый прогресс в улучшении методов хирургического лечения БИК, частота рецидивов и неэффективности операции остается высокой: описано до 15\% случаев микроаденом и до 50\% случаев макроаденом с отсутствием стойкой ремиссии, что делает вопрос медикаментозной терапии БИК особенно актуальным [51]. В настоящее время наиболее часто в лечении БИК у взрослых пациентов используются ингибиторы синтеза кортизола (кетоконазол, митотан, метирапон) или антагонисты ГкР (мифепристон), которые снижают уровень кортизола или блокируют его действие на периферии, но не влияют на причину заболевания [52, 53]. Ни у одного из вышеперечисленных препаратов нет показаний к применению при БИК у детей.

В данной статье мы остановимся на потенциальных возможностях таргетной терапии БИК. 


\section{Аналог соматостатина}

По многочисленным иммуногистохимическим исследованиям в кортикотропиномах определяется высокая экспрессия соматостатиновых рецепторов 5 и 3 типа [52]. Как было представлено выше, в кортикотропиномах, несущих мутацию в гене USP8, выявляются соматостатиновые рецепторы (ССТР) 5 типа [28]. Пасиреотид, являясь аналогом соматостатина, связывается с ССТР5, приводя к ингибированию секреции АКТГ, что и сделало его применение при БИК достаточно эффективным [11, 52, 54]. Клинический эффект на фоне терапии достигался у $40 \%$ пациентов в виде снижения уровня кортизола в суточном анализе мочи до нормальных значений, компенсации клинических проявлений и уменьшения размеров кортикотропином; из побочных эффектов, помимо гастроинтестинальных, в большинстве случаев (до 70-80\%) отмечалось развитие тенденции к гипергликемии, потребовавшее назначения сахароснижающей терапии [11, 52].

\section{Агонисты дофаминовых рецепторов}

Учитывая высокую экспрессию $\mathrm{D}_{2}$-рецепторов в ткани кортикотропином, проводились исследования по эффективности терапии каберголином при БИК, которые не показали высокой эффективности при монотерапии: только у 20-40\% пациентов отмечалось лабораторное улучшение показателей $[11,52]$. Дозы каберголина, используемые при БИК, превышают терапевтические дозы при лечении пролактином и могут достигать 5-7 мг/неделю. В связи с высокой частотой экспрессии и ССТР 2 и 5 типа, и $\mathrm{D}_{2}$-рецепторов в ткани кортикотропином в настоящее время проводятся испытания комбинированной терапии при БИК [52, 54].

\section{Алкилирующие средства}

Темозоломид применяется при новообразованиях ЦНС, так как проникает через гематоэнцефалический барьер. Данный препарат индуцирует клеточный апоптоз путем метилирования ДНК. Клинический эффект

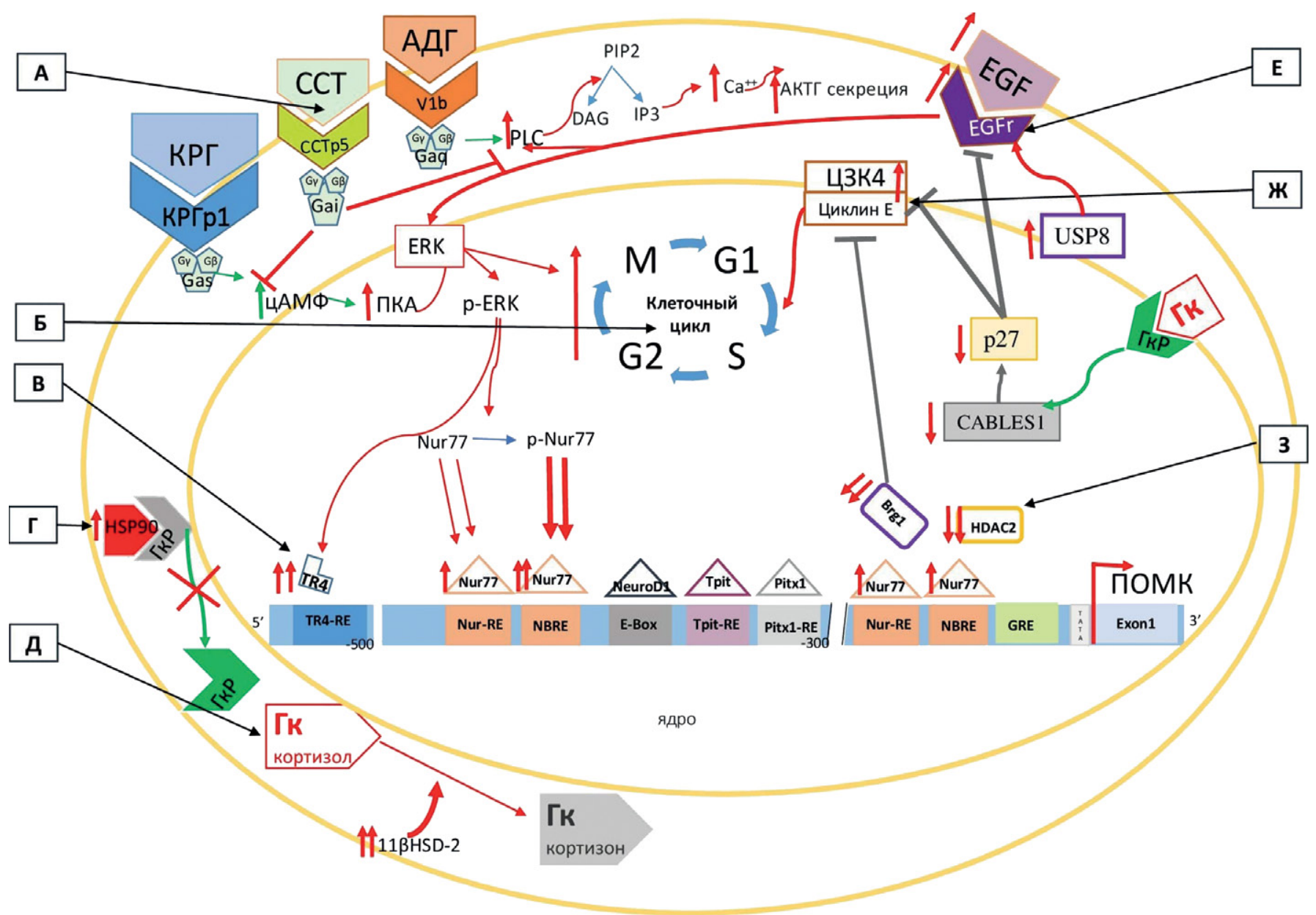

Рисунок 3. Перспективы таргетной терапии при болезни Иценко-Кушинга.

A:Пасиреотид связывается с соматостатиновыми рецепторами 5 типа (ССТР5) и приводит к снижению секреции адренокортикотропного гормона (АКТГ). Б: Темозоламид индуцирует клеточный апоптоз путем метилирования ДНК.

B: Биниметиниб, ингибитор митоген-активируемых протеинкиназ МЕК-162, снижает транскрипцию тестикулярного ядерного рецептора 4 типа (TR4) и снижает экспрессию гена РОМС.

Г: Силибинин ингибирует белок теплового шока 90 (HSP90), который участвует в регуляции глюкокортикоидного рецептора (ГкР) и развитии резистентности к механизму обратной отрицательной связи.

Д: Кетоконазол ингибирует 11-бета-гидроксилазу, катализирующую образование кортизола из 11-дезоксикортизола.

Е: Гефитиниб селективно ингибирует тирозинкиназу рецепторов эпидермального ростового фактора (EGF).

Ж: Росковитин, ингибитор циклинзависимой киназы (CDK), угнетает выработку ПОМК и регулирует клеточный рост.

3: Вориностат, ингибитор гистон-деацетилазы (HDAC2), обладает способностью индуцировать апоптоз и ингибировать синтез АКтГ.

Изображения, представленные на рисунках: Т указывают на активирующее влияние Т указывают на ингибирующее влияние 
терапии развивается в период до 2 мес, частичный или полный ответ на терапию отмечается до 80\% пациентов с БИК [55]. Темозоломид может применяться как в качестве монотерапии, так и в комбинации с пасиреотидом.

\section{Ингибиторы рецептора EGF}

Гефитиниб является селективным ингибитором тирозинкиназы рецепторов EGF и широко применяется при метастатическом немелкоклеточном раке легкого. В связи с выявлением участия рецептора EGF в патогенезе развития кортикотропином, в том числе и при мутациях в гене USP8, проводятся исследования ингибиторов рецептора EGF в терапии БИК [52].

\section{Ингибитор циклин-зависимой киназы}

Росковитин, являясь ингибитором CDK, оказывает ингибирующее влияние на выработку ПОМК и регулирует клеточный рост [20]. На фоне терапии росковитином у пациентов с кортикотропиномами было отмечено значимое снижение уровня АКТГ и умеренный ингибирующий эффект на рост аденомы [52].

\section{Другие возможные таргетные препараты}

Силибинин обладает ингибирующим действием на белок теплового шока 90, который участвует в регуляции ГкР и развитии резистентности к механизму обратной отрицательной связи при кортикотропиномах [52]. В настоящее время ведутся активные исследования этого препарата при кортикотропиномах и других новообразованиях.

Ингибитор гистон-деацетилазы - вориностат также обладает способностью индуцировать апоптоз и ингибировать синтез АКТГ [52].

Сигнальный путь митоген-активируемых протеинкиназ (МАРК) участвует в инициировании транскрипции тестикулярного рецептора 4 типа (TR4); применение ингибитора митоген-активируемых протеинкиназ МЕК-162 (биниметиниб) приводит к снижению транскрипции TR4 и, как следствие, снижает экспрессию ПОМК [56].

Механизмы действия таргетной терапии при БИК представлены на рис. 3.

\section{ЗАКЛЮЧЕНИЕ}

БИК - крайне редкое для педиатрической практики, тяжелое мультисистемное заболевание.

В большинстве случаев БИК - спорадическое заболевание, обусловленное соматическими мутациями, нарушающими механизмы регуляции гипоталамо-гипофизарно-надпочечниковой оси и работы клеточного цикла кортикотрофов.

В то же время нужно учитывать возможность появления кортикотропином в составе множественных эндокринных неоплазий, что может значимо повлиять на тактику ведения и наблюдения пациентов в динамике.

Вероятность неэффективного оперативного лечения БИК делает перспективным развитие таргетной терапии, направленной на ингибирование механизмов гиперпродукции АКТГ и клеточной пролиферации.

\section{ДОПОЛНИТЕЛЬНАЯ ИНФОРМАЦИЯ}

Источник финансирования. Поисково-аналитическая работа по подготовке рукописи проведена в рамках исполнения государственного задания № АААА-А20-120011790183-5.

Конфликт интересов. Все авторы декларируют отсутствие явных и потенциальных конфликтов интересов, связанных с публикацией настоящей статьи.

Участие авторов. Все авторы одобрили финальную версию статьи перед публикацией, выразили согласие нести ответственность за все аспекты работы, подразумевающую надлежащее изучение и решение вопросов, связанных с точностью или добросовестностью любой части работы.

\section{СПИСОК ЛИТЕРАТУРЫ | REFERENCES}

1. Sharma ST, Nieman LK, Feelders RA. Cushing's syndrome: epidemiology and developments in disease management. Clin Epidemiol. 2015;7:281-293. Published 2015 Apr 17. doi: 10.2147/CLEP.S44336

2. Stratakis CA. An update on Cushing syndrome in pediatrics. Ann Endocrinol (Paris). 2018;79(3):125-131. doi: 10.1016/j.ando.2018.03.010

3. Storr HL, Savage MO. Management of endocrine disease: Paediatric Cushing's disease. Eur J Endocrinol. 2015;173(1):R35-R45. doi: 10.1530/EJE-15-0013

4. Pivonello R, De Leo M, Cozzolino A, Colao A. The Treatment of Cushing's Disease. Endocr Rev. 2015;36(4):385-486. doi: 10.1210/er.2013-1048

5. Лапшина А.М., Абросимов А.Ю., Марова Е.И. Клиническая и морфофункциональная характеристика опухолей, продуцирующих адренокортикотропный гормон // Проблемы эндокринологии. — 2008. Т. 54. — №4. — С. 49-54. Веб. 7 дек. 2020. [Lapshlna AM, Abrosimov AYu, Marova YI. The clinical and morphofunctional characteristics of adrenocorticotropic hormoneproducing tumors. Problems of Endocrinology. 2008;54(4):49-54 (In Russ.)]. doi: 10.14341/probl200854449-54.

6. Maira M, Couture C, Le Martelot G, et al. The T-box factor Tpit recruits SRC/p160 co-activators and mediates hormone action. J Biol Chem. 2003;278(47):46523-32. doi: 10.1074/jbc.m305626200.

7. Du L, Bergsneider M, Mirsadraei L, et al. Evidence for orphan nuclear receptor TR4 in the etiology of Cushing disease. Proceedings of the National Academy of Sciences of the United States of America. 2013 May;110(21):8555-8560. doi: 10.1073/pnas.1306182110.

8. Kirschke E, Goswami D, Southworth D, et al. Glucocorticoid receptor function regulated by coordinated action of the Hsp90 and Hsp70 chaperone cycles. Cell. 2014;157(7):1685-1697. doi: 10.1016/j.cell.2014.04.038

9. Bilodeau S, Vallette-Kasic S, Gauthier Y, et al. Role of Brg1 and HDAC2 in GR trans-repression of the pituitary POMC gene and misexpression in Cushing disease. Genes Dev. 2006;20(20):2871-2886. doi: 10.1101/gad.1444606

10. Albani A, Theodoropoulou M, Reincke M. Genetics of Cushing's disease. Clin Endocrinol (Oxf). 2018;88(1):3-12. doi: 10.1111/cen.13457

11. Tritos NA, Biller BMK. Medical Management of Cushing Disease. Neurosurg Clin NAm. 2019;30(4):499-508. doi: 10.1016/j.nec.2019.05.007

12. Herman V, Fagin J, Gonsky R, et al. Clonal origin of pituitary adenomas. J Clin Endocrinol Metab. 1990;71(6):1427-1433. doi: 10.1210/jcem-71-6-1427

13. Hernández-Ramírez LC, Stratakis CA. Genetics of Cushing's Syndrome. Endocrinol Metab Clin North Am. 2018;47(2):275-297. doi: 10.1016/j.ecl.2018.02.007

14. Huizenga NA, de Lange P, Koper JW, et al. Human adrenocorticotropinsecreting pituitary adenomas show frequent loss of heterozygosity at the glucocorticoid receptor gene locus. J Clin Endocrinol Metab. 1998;83(3):917-921. doi: 10.1210/jcem.83.3.4648

15. Tateno T, Izumiyama $\mathrm{H}$, Doi $\mathrm{M}$, et al. Differential gene expression in ACTH -secreting and non-functioning pituitary tumors. Eur J Endocrinol. 2007;157(6):717-724. doi: 10.1530/EJE-07-0428

16. Evang JA, Borota OC, Melum E, et al. HDAC2 expression and variable number of repeats in exon 1 of the HDAC2 gene in corticotroph adenomas. Clin Endocrinol (Oxf). 2010;73(2):229-235. doi: 10.1111/j.1365-2265.2010.03805.x 
17. Tabuchi Y, Kitamura T, Fukuhara A, et al. Nur77 gene expression levels were involved in different ACTH-secretion autonomy between Cushing's disease and subclinical Cushing's disease. Endocr J. 2016;63(6):545-554. doi: 10.1507/endocrj.EJ15-0695

18. Korbonits M, Bujalska I, Shimojo M, et al. Expression of 11 betahydroxysteroid dehydrogenase isoenzymes in the human pituitary: induction of the type 2 enzyme in corticotropinomas and other pituitary tumors. J Clin Endocrinol Metab. 2001;86(6):2728-2733. doi: 10.1210/jcem.86.6.7563

19. Wells AD, Morawski PA. New roles for cyclin-dependent kinases in T cell biology: linking cell division and differentiation. Nat Rev Immunol. 2014;14(4):261-270. doi: 10.1038/nri3625

20. Jordan S, Lidhar K, Korbonits M, et al. Cyclin D and cyclin E expression in normal and adenomatous pituitary. Eur J Endocrinol. 2000;143(1):R1-R6. doi: 10.1530/eje.0.143r001

21. Roussel-Gervais A, Bilodeau S, Vallette S, et al. Cooperation between cyclin E and p27(Kip1) in pituitary tumorigenesis. Mol Endocrinol. 2010;24(9):1835-1845. doi: 10.1210/me.2010-0091

22. Roussel-Gervais A, Couture C, Langlais D, et al. The Cables1 Gene in Glucocorticoid Regulation of Pituitary Corticotrope Growth and Cushing Disease. J Clin Endocrinol Metab. 2016;101(2):513-522. doi: 10.1210/jc.2015-3324

23. Hernández-Ramírez LC, Gam R, Valdés N, et al. Loss-offunction mutations in the CABLES1 gene are a novel cause of Cushing's disease. Endocr Relat Cancer. 2017;24(8):379-392. doi: 10.1530/ERC-17-0131

24. Theodoropoulou M, Arzberger T, Gruebler Y, et al. Expression of epidermal growth factor receptor in neoplastic pituitary cells: evidence for a role in corticotropinoma cells. J Endocrinol. 2004;183(2):385-394. doi: 10.1677/joe.1.05616

25. Qian ZR, Sano T, Asa SL, et al. Cytoplasmic expression of fibroblast growth factor receptor-4 in human pituitary adenomas: relation to tumor type, size, proliferation, and invasiveness. J Clin Endocrino Metab. 2004;89(4):1904-1911. doi: 10.1210/jc.2003-031489

26. Reincke M, Sbiera S, Hayakawa A, et al. Mutations in the deubiquitinase gene USP8 cause Cushing's disease. Nat Genet 2015;47(1):31-38. doi: 10.1038/ng.3166

27. Renner U, Ciato D, Stalla GK. Recent advances in understanding corticotroph pituitary tumor initiation and progression. F1000Res. 2018;7:F1000 Faculty Rev-1354. Published 2018 Aug 29. doi: 10.12688/f1000research.14789.1

28. Hayashi K, Inoshita N, Kawaguchi K, et al. The USP8 mutational status may predict drug susceptibility in corticotroph adenomas of Cushing's disease. Eur J Endocrinol. 2016;174(2):213-226. doi: 10.1530/EJE-15-0689

29. Faucz FR, Tirosh A, Tatsi C, et al. Somatic USP8 Gene Mutations Are a Common Cause of Pediatric Cushing Disease. J Clin Endocrinol Metab. 2017;102(8):2836-2843. doi: 10.1210/jc.2017-00161

30. Cohen M, Persky R, Stegemann R, et al. Germline USP8 Mutation Associated With Pediatric Cushing Disease and Other Clinical Features: A New Syndrome. J Clin Endocrinol Metab. 2019:104(10):4676-4682. doi: 10.1210/jc.2019-00697

31. Buckley N, Bates AS, Broome JC, et al. p53 Protein accumulates in Cushings adenomas and invasive non-functiona adenomas. J Clin Endocrinol Metab. 1994;79(5):1513-1516. doi: 10.1210/jcem.79.5.7962351

32. Tadjine M, Lampron A, Ouadi L, Bourdeau I. Frequent mutations of beta-catenin gene in sporadic secreting adrenocortical adenomas. Clin Endocrinol (Oxf). 2008;68(2):264-270. doi: 10.1111/j.1365-2265.2007.03033.x

33. Murat CB, Braga PB, Fortes MA, et al. Mutation and genomic amplification of the PIK3CA proto-oncogene in pituitary adenomas. Braz J Med Biol Res. 2012;45(9):851-855. doi: 10.1590/s0100-879x2012007500115

34. Simonds WF, Varghese S, Marx SJ, Nieman LK. Cushing's syndrome in multiple endocrine neoplasia type 1. Clin Endocrinol (Oxf). 2012;76(3):379-386. doi: 10.1111/j.1365-2265.2011.04220.x

35. de Laat JM, Dekkers OM, Pieterman CR, et al. Long-Term Natural Course of Pituitary Tumors in Patients With MEN1: Results From the DutchMEN1 Study Group (DMSG). J Clin Endocrinol Metab. 2015;100(9):3288-3296. doi: 10.1210/JC.2015-2015

36. Bilezikjian LM, Vale WW. The Local Control of the Pituitary by Activin Signaling and Modulation. Open Neuroendocrinol J. 2011;4:90-101. doi: 10.2174/1876528901104010090

37. Trouillas J, Labat-Moleur F, Sturm N, et al. Pituitary tumors and hyperplasia in multiple endocrine neoplasia type 1 syndrome
(MEN1): a case-control study in a series of 77 patients versus 2509 non-MEN1 patients. Am J Surg Pathol. 2008;32(4):534-543. doi: 10.1097/PAS.0b013e31815ade45

38. Chasseloup F, Pankratz N, Lane J, et al. Germline CDKN1B Loss-ofFunction Variants Cause Pediatric Cushing's Disease With or Without an MEN4 Phenotype. J Clin Endocrinol Metab. 2020;105(6):1983-2005. doi: 10.1210/clinem/dgaa160

39. Steiner AL, Goodman AD, Powers SR. Study of a kindred with pheochromocytoma, medullary thyroid carcinoma, hyperparathyroidism and Cushing's disease: multiple endocrine neoplasia, type 2. Medicine (Baltimore). 1968;47(5):371-409. doi: 10.1097/00005792-196809000-00001

40. Kasturi K, Fernandes L, Quezado M, et al. Cushing Disease in a patient with Multiple Endocrine Neoplasia type 2B. J Clin Trans/ Endocrinol Case Rep. 2017;4:1-4. doi: 10.1016/j.jecr.2017.02.001

41. Naziat A, Karavitaki N, Thakker R, et al. Confusing genes: a patient with MEN2A and Cushing's disease. Clin Endocrinol (Oxf). 2013;78(6):966-968. doi: 10.1111/cen.12072

42. Daly AF, Jaffrain-Rea ML, Ciccarelli A, et al. Clinical characterization of familial isolated pituitary adenomas. J Clin Endocrinol Metab. 2006;91(9):3316-3323. doi: 10.1210/jc.2005-2671

43. Beckers A, Aaltonen LA, Daly AF, Karhu A. Familial isolated pituitary adenomas (FIPA) and the pituitary adenoma predisposition due to mutations in the aryl hydrocarbon receptor interacting protein (AIP) gene. Endocr Rev. 2013;34(2):239-277. doi: 10.1210/er.2012-1013

44. Song MS, Rossi JJ. Molecular mechanisms of Dicer: endonuclease and enzymatic activity. Biochem J. 2017;474(10):1603-1618. Published 2017 May 4. doi: 10.1042/BCJ20160759

45. Lania A, Mantovani G, Spada A. CAMP pathway and pituitary tumorigenesis. Ann Endocrinol (Paris). 2012;73(2):73-75. doi: 10.1016/j.ando.2012.03.027

46. Correa R, Salpea P, Stratakis CA. Carney complex: an update. Eur J Endocrinol. 2015;173(4):M85-M97. doi: 10.1530/EJE-15-0209

47. Kirschner LS, Carney JA, Pack SD, et al. Mutations of the gene encoding the protein kinase A type I-alpha regulatory subunit in patients with the Carney complex. Nat Genet. 2000;26(1):89-92. doi: 10.1038/79238

48. Kiefer FW, Winhofer $Y$, lacovazzo D, et al. PRKAR1A mutation causing pituitary-dependent Cushing disease in a patient with Carney complex. Eur J Endocrinol. 2017;177(2):K7-K12. doi: 10.1530/EJE-17-0227

49. Pack SD, Qin LX, Pak E, et al. Common genetic changes in hereditary and sporadic pituitary adenomas detected by comparative genomic hybridization. Genes Chromosomes Cancer. 2005;43(1):72-82. doi: 10.1002/gcc.20162

50. Hernández-Ramírez LC, Tatsi C, Lodish MB, et al. Corticotropinoma as a Component of Carney Complex. J Endocr Soc. 2017;1(7):918-925 Published 2017 May 30. doi: 10.1210/js.2017-00231

51. Petersenn S, Beckers A, Ferone D, et al. Therapy of endocrine disease: outcomes in patients with Cushing's disease undergoing transsphenoidal surgery: systematic review assessing criteria used to define remission and recurrence. Eur J Endocrinol. 2015;172(6):R227-R239. doi: 10.1530/EJE-14-0883

52. Theodoropoulou M, Reincke M. Tumor-Directed Therapeutic Targets in Cushing Disease. J Clin Endocrinol Metab. 2019;104(3):925-933. doi: 10.1210/jc.2018-02080

53. Марова Е.И., Арапова С.Д. Современные возможности лечения болезни Иценко-Кушинга // Эффективная фармакотерапия. 2010. — № 37. - C. 54-60. [Marova E.I., Arapova S.D. Sovremennye vozmozhnosti lechenija bolezni Icenko-Kushinga. Jeffektivnaja farmakoterapija. 2010(37):54-60. (In Russ.)].

54. Лапшина А.М. Клиническая и морфофункциональная характеристика опухолей, продуцирующих адренокортикотропный гормон. Дисс. ... ВАК РФ 14.00 .03 канд. мед. наук. - М.; 2009. [Lapshina A.M. Klinicheskaja i morfo-funkcional'naja harakteristika opuholej, producirujushhih adrenokortikotropnyj gormon. Diss. ... VAK RF 14.00.03 kand. med. nauk. — M.; 2009]. Доступно по: https://www.dissercat.com/ content/klinicheskaya-i-morfo-funktsionalnaya-kharakteristikaopukholei-produtsiruyushchikh-adrenoko. Ссылка активна на 07.12.2020.

55. Ji Y, Vogel RI, Lou E. Temozolomide treatment of pituitary carcinomas and atypical adenomas: systematic review of case reports. Neurooncol Pract. 2016;3(3):188-195. doi: 10.1093/nop/npv059

56. Zhang D, Bergsneider M, Wang MB, Heaney AP. Targeting the ERK pathway for the treatment of Cushing's disease. Oncotarget. 2016;7(43):69149-69158. doi: 10.18632/oncotarget.12381 
Рукопись получена: 24.09.2019. Одобрена к публикации: 11.12.2020. Опубликована online: 30.12.2020.

\section{ИНФОРМАЦИЯ ОБ АВТОРАХ [AUTHORS INFO]}

*Янар Эда Альперовна [Eda А. Yanar]; адрес: Россия, 117036, Москва, ул. Дм. Ульянова, д. 11 [address:

11 Dm. Ulyanova street, 117036 Moscow, Russia]; ORCID: https://orcid.org/0000-0002-8610-821X; eLibrary SPIN: 3104-6767; e-mail: edayanar@mail.ru

Маказан Надежда Викторовна, к.м.н. [Nadezhda V. Makazan, MD, PhD]; ORCID: https://orcid.org/0000-0003-3832-6367; eLibrary SPIN: 7156-6517; e-mail: Nmakazan@ya.ru

Орлова Елизавета Михайловна, д.м.н., доцент [Elizaveta M. Orlova, MD, Associate Professor];

ORCID: https://orcid.org/0000-0001-6196-5322; eLibrary SPIN: 5221-4235; e-mail: elizaveta.orlova@mail.ru

Карева Мария Андреевна, д.м.н. [Maria A. Kareva, MD]; ORCID: https://orcid.org/0000-0003-1320-6561;

eLibrary SPIN: 5089-0310; e-mail: i_marusya@mail.ru

\section{ЦИТИРОВАТЬ:}

Янар Э.А., Маказан Н.В., Орлова Е.М., Карева М.А. Молекулярно-генетические основы болезни Иценко-Кушинга у детей и перспективы таргетной терапии // Проблемы эндокринологии. — 2020. — Т. 66. — №6. — С. 39-49. doi: https://doi.org/10.14341/probl12676

\section{TO CITE THIS ARTICLE:}

Yanar EA, Makazan NV, Orlova EM, Kareva MA. Genetic basis of Cushing's disease in children and targeted therapeutic future perspectives. Problems of Endocrinology. 2020;66(6):39-49. doi: https://doi.org/10.14341/probl12676 